Volume 9, No.4, July - August 2020

International Journal of Advanced Trends in Computer Science and Engineering

Available Online at http://www.warse.org/IJATCSE/static/pdf/file/ijatcse136942020.pdf

https://doi.org/10.30534/ijatcse/2020/136942020

\title{
An Efficient Congestion Aware Multipath Routing Protocol using Oppositional Artificial Flora Algorithm in Mobile Adhoc Networks
}

\author{
R. Jayaraj ${ }^{1}$, Dr. T. Suresh ${ }^{2}$, Dr. K. B Jayaraman ${ }^{3}$ \\ ${ }^{1}$ Research Scholar, Department of CSE, Annamalai University, TamilNadu, India,India.jthunder137@ gmail.com \\ ${ }^{2}$ Associate Professor, Department of CSE, Annamalai University, TamilNadu, India \\ sureshaucse@gmail.com \\ ${ }^{3}$ Professor, Dean Academics, Sri Venkateshwara College of Engineering and Technology, Pondicherry \\ annaijayaraman@yahoo.com
}

\begin{abstract}
Mobile adhoc networks (MANET) comprises a collection of mobile nodes, which poses several issues like maximum error rate, minimum bandwidth, dynamic nature of mobile nodes makes it difficult to ensure quality of service (QoS). For improving of TCP performance of MANET, this paper presents an effective TCP congestion control mechanism. At the same time, QoS aware multipath routing problem in MANET has received more attention among researchers. Multimedia applications like audios and videos have high QoS needs in MANET and it leads to complex routing protocol with many QoS constraints. In QoS routing, a fundamental issue is the identification of the route which fulfils many QoS constraints. At the same time, congestion, mobility, and packet loss in dynamic topology of network results in degraded QoS performance. To resolve these issues, in this paper, a new QoS based Congestion Aware Multipath Routing Protocol using Oppositional Artificial Flora (OAF) algorithm, called QCMRP-OAF is presented. The inclusion of oppositional based learning (OBL) mechanism in artificial flora (AF) algorithm helps to improve the convergence rate and exploration process. Besides, the proposed QCMRP-OAF algorithm derives a new fitness function for route selection using energy, link lifetime, congestion, and distance. The proposed QCMRP-OAF algorithm has been simulated using network simulator (NS 2.5) tool and the results are examined under several aspects. The experimental outcome indicated that the presented QCMRP-OAF algorithm is superior to other methods interms of energy, throughput, delay, packet loss and congestion level.
\end{abstract}

Key words: Artificial Flora, Congestion Control, MANET, Multipath Routing, Oppositional based learning

\section{INTRODUCTION}

Mobile adhoc network (MANET) is defined as a multi-hop transitory independent model which is made up of mobile nodes by a wireless connection. The nodes present in MANET are capable of joining dynamically and leave from the system
[1]. The rising familiarity of MANET necessitate maximum data rate and QoS ensured traffic representing that the wireless connections acts as a major part in future internetworks. Reliable transport protocols like TCP (transmission control protocol) can be tuned to perform well in classical wired network, where packet loss occurs because of congestion. At the same time, a network with wireless links with the characteristics appears due to congestion. However, networks with wireless links with features of maximum bit error rate, low bandwidth, high delay, frequent mobility, etc suffer from significant loss because of bit error and handoff. As it is very flexible and lagging in proper architecture, it is extensively applied in armed forces as well as civilian sectors. The main aim of MANET is to build a communication among rescuers, searching communities and healthcare personnel in natural disaster management in which network structure has been failed. Also, it is applied in routine activities such as habitation observation, ecological tracking as well as ocean monitoring, and several other evolving domains like traveller location, destination monitoring, social network etc.

Ad hoc wireless networks are the type of infrastructure-less system where massive tools are comprised with wireless communication as well as networking ability with routing potentials in mobility. These systems do not contain fixed topologies as covering enormous region. Such topologies might be tailored in a dynamic manner and suddenly even at mobility. Conventional routing protocols are employed for internet centric wireless system where it is not applied directly to MANET, as few considerations are invalid for dynamic based networks. The bandwidth accessibility is a mandatory problem engaged in ad hoc networks which leads to cause many problems while designing routing protocols, in which every node involves routing by transmitting data according to the network connection. The network applies wireless channel for communication, and it is influenced by propagation loss, shadow reduction, as well as multipath Rayleigh fading.

Some of the tools contributed in MANETs must be capable of detecting the existence of alternate devices and process the 
essential tasks which provide communication, data distribution and many other services. Hence, because of the restricted bandwidth and shares common channel for every node, then a problem is emerged named as congestion which is highly complicated in wireless MANET. Congestion is often assumed to be the major factor that reduces the network performance and results in packet losses, bandwidth fading, maximum time, and energy application by simulating congestion recovery models. Diverse methods are deployed for reducing the congestion and to enhance the ability of wireless MANET. There are few protocols used for transmitting nodes regarding the recent state of network congestion and guides in re-routing the transmissions on the basis of congestion levels and protocols applied.

A wireless MANET were developed rapidly and defined as one of the major systems that depend upon a self-organized and easily deployable module. As mentioned above, it is highly reliable and inspired by many developers in which networks topology is modified rapidly. Though it has maximum available resources, it is still filled with some limitations that are resolved using several studies. The major disadvantages of MANETs are minimum bandwidth, battery power, processing energy, and trust. Many studies have been presented to solve these constraints on MANETs. Some of them are routing, Congestion control models, congestion adaptive methods, load balancing, and privacy. Among others, the most wanted congestion adaptive methods for MANETs are assumed to enhance the system performance. The main aim of MANET is developing routing protocols which equalizes dynamics of ad hoc networks.

In [2], a distance vector mechanism named as ad hoc on demand distance vector (AODV), were projected that is an on-demand route acquisition approach; nodes which are not placed on active paths does not retain the routing data or participating in periodic routing table interchanging. Furthermore, [3] has developed Dynamic Source Routing (DSR) method for the purpose of increasing the function, for instance, to enable scaling of massive networks and inclusion of novel features to protocol, like multicast routing, QoS conservations as well as resource controlling. In [4], a creative model such as Dynamic Destination-Sequenced Distance-Vector Routing (DSDV), which is provided when computers are applied as routers, and utilized for sending packets whenever required. This method has been applied in network layer 3 and over the MAC layer software in layer 2.

In [5], a novel shared routing protocol, WRP is proposed for a packet radio network that is operated based on second-to-final hop node to target. A routing associated with congestion awareness as well as MANET application has been employed. It is mainly applied to eliminate the congestion in first place and should to be applicable. Each node in the route requests its consecutive node with maximum chance of occurring congestion. The existing node employs a "bypass" route for bypassing the capable congestion region to first non-congested node on primary route. Traffic is divided possibility into 2 routes namely, primary and bypass, which significantly reduces the opportunity of existence of congestion. In [6], an Efficient Congestion Adaptive Routing Protocol (ECARP) for MANETs was projected that performs an outstanding function than alternate routing protocols even at heavy traffic loads. The main aim of this method is to confirm the maximum accessibility of other routes and limit the expired route. This can be accomplished by enhancing the parameters involved in routing protocols which generally utilizes maximum duration for connection recovery. The count of packets presents in buffer which is used for computing the state of congestion.

In [7], a CARP for mobile ad hoc networks (CARM) were deployed and applies retransmission number weighted channel delay as well as buffer queuing delay with priority for minimum congested and high throughput links to enhance the channel consumption. A congestion adaptive AODV (CAAODV) routing protocol was introduced to stream the video in MANET which is mainly developed for multimedia domains. As video data is highly delicate in delay and packet loss, the determination of congestion is based on maximum packet delivery time (PDT) and packet delivery ratio (PDR). In [8], a CARP system is utilized to reactive ad hoc routing protocol, which is implied as CAAODV routing protocol. The major features of this approach are identifying another route, while the congestion occurs in primary route, according to the condition of buffer size of adjacent node and state of buffer size of consecutive node on primary route.

In [9], a hop- by-hop CARP have been established by integrating weight value and routing metric, on the basis of data value, queuing delay, link superiority, and MAC overload in benchmark cost function for congestion level. Because of the channel interference, multipath improves the delay and ineffective in congested networks. In [10], a CA multipath dynamic source routing protocol (CAWMP-DSR) was presented for greater number of node disjoint paths under the application of multipath DSR. A collection of disjoint multipath are produced and manages the issue of end-to-end delay with the help of correlation factor as well as end-to-end delay is enhanced. In [11], actual DSR protocol was changed to describe the action of congestion by tracking and addressing various resource application thresholds as QoS parameters and applies multipath routing as well as load balancing at the time of using QoS in MANETs for CBR multimedia. Here, the battery level and queue length were applied as major resource parameters.

In [12], developers have defined protocols for all-to-all dissemination in MANET. It calculates the function of GOSSIP3 dissemination protocol under different network overheads ended up with MAC layer congestion awareness is one of the vital factors to enhance the performance efficiency. In [13], CARM was presented, where it applies the retransmission number weighted channel delay as well as 
buffer queuing delay, by preferring lower congested maximum throughput connections to increase channel application. In [14], AODV (CA-AODV) routing protocol was developed for video streaming in MANET which offers various non-congested path when the nodes are congested. A hop-by- hop CARP were established; but it was stimulated to congestion adaptivity.

In [15], a work load oriented model was depicted that assume the workload of path and network to find a route with minimum congestion, and applicable for minimum load. An ECARP is projected for MANETs which assure the maximum accessibility of another routes. [16] presented a multipath routing with load balancing; however, it does not assume the variable congestion state of network. In CARP models are demonstrated and identified the parameters with similar protocol that tends to discover the solution by assuming load balancing and CA approach in the newly developed model.

Since congestion, mobility, and packet loss in dynamic topology of network leads to reduced QoS results, this paper introduces a new QoS based Congestion Aware Multipath Routing Protocol using Oppositional Artificial Flora (OAF) algorithm, called QCMRP-OAF. The application of oppositional based learning (OBL) mechanism in artificial flora $(\mathrm{AF})$ algorithm helps to increase the convergence rate and exploration process. In addition, a new fitness function is also derived in QCMRP-OAF algorithm for route selection using energy, link lifetime, congestion, and distance. The proposed QCMRP-OAF algorithm has been simulated using network simulator (NS 2.5) tool and the results are examined under several aspects.

\section{THE PROPOSED QCMRP-OAF ALGORITHM}

The working principle of the QCMRP-OAF model for congestion aware multipath selection is depicted in Figure 1. At the earlier stage, the mobile nodes in MANET undergo initialization in a particular target region. Next, the routes between any of the two nodes are identified. At last, the multiple routes are chosen by the QCMRP-OAF algorithm and derived FF. These processes are discussed in the following subsections.

\subsection{Initialization Phase}

Principally, the mobile nodes in the MANET undergo deployment and initialization in a target area. Then, a connection is established between the mobile nodes in the network. The coordinate points of the mobile nodes are determined in which the position and velocity of the nodes could be determined in the future. Assume $m$ be the node count in MANET, where $1<i<m$.

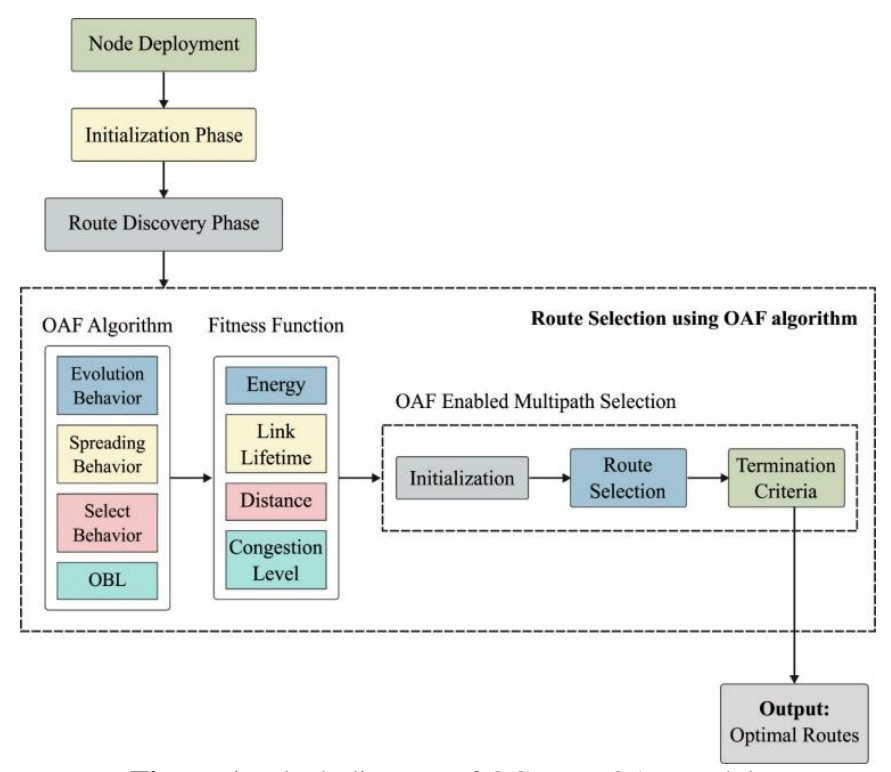

Figure 1: Block diagram of QCMRP-OAF model

\subsection{Route Discovery Phase}

In the second phase, route discovery process will begin where the routes among source and destination nodes are explored. The route between two nodes is based on the link count presented among the nodes linked to corresponding communication channel. The path discovery is the second step in the proposed multipath selection scheme [17]. Consider R be the route count exist among two nodes, where $1<j<R$. In general, the communication channel between the nodes is based on the links connected to the channel and several paths might exist. Consider $S$ and $D$ are the source and destination mobile nodes. The data transmission from $\mathrm{S}$ to $\mathrm{D} S$ and $D$ is carried out using several routes exists. In case of $m$ number of nodes present exist among $S$ and $D, \mathrm{~K}$ be the explored routes for $\mathrm{L}$ links exist in the nodes linked to the communication channel.

\subsection{Route Selection using OAF algorithm}

\section{A. Proposed OAF algorithm}

Generally, Plants have various forms to disperse seed. Seed diffusion is split into autochory and allochory [18]. The former one defines the self-dispersal of seeds, and the latter one indicates dispersal of seeds by means of external agents. For instance, the physical dispersal is autochory, and natural dispersal, anemochory, and hydrochory comes under allochory. Autochory provide the circumstances for plants to transfer to a more proper atmosphere freely. Melons, sorrels and impatiens multiply seeds through Autochory. When a sorrel is mature, then fruit would be loculicidal, while shells would be coil up to burst the seed. The fruits of the impatiens will burst open and diffuse the seeds surrounding it. As soon as a melon attains a definite ripeness, the seeds will be separated with mucus generated from the peak of a melon. The space could be $5 \mathrm{~m}$. However, allochory gives migrating state to farther and uncultivated sections. For example, the dispersion path and distance between the seeds are calculated 
using wind in anemochory as the wind speed and direction might be changed frequently. Such dispersal broadens make an extraordinary study about flora and reduce the deterioration of the forest.

Due to unfavorable natural atmosphere or struggle, climate change, or the distribution region of flora could be migrated, prolonged or minimized. Since flora emigrates to fresh atmosphere, the characteristics of flora will grow as well. So, the emigration of flora could modify dispersion region and encourage the growth, rebirth and extinct of flora. Plants are static and brainless, whereas flora can discover their suitable surrounding to survive through dispersal of seeds and reproduction. During emigration and reproduction of flora, the actual plant spread out seeds randomly on all sides in a definite region. The survival chance of a seed is dissimilar with respect to the outer atmosphere.

In a good atmosphere, a flora remains and reproduce seeds more or less once being grown. In bad atmosphere, plant may develop by adapting the atmosphere or may wipe out. Allochory propagate possible ways that the plants perhaps reproduce in other place as soon as the flora is fully destroyed in this region. The seeds might be taken to any fresh place anywhere and continue its reproduction. The flora would transfer to a better place by multi-generational dispersal. In the process of migration and reproduction, the plant finishes the effort of identifying the better environment through the extinction, evolution and rebirth of plant. The AF algorithm is composed of 4 fundamental components. Initially, Original plants defines that the plants which are likely to distribute the seeds. Secondly, Offspring plants are said to be developed from seeds of original plants which is not able to spread seeds at the same time. Third, Plant location describes the plant location. Finally, Propagation distance shows how far the seeds can be distributed. It is operated on 3 main behavioral patterns. The Evolution behavior is that the possible evolving time taken by plants to be adapted to the atmosphere. Then, spreading natures means the action of seeds, it moves by autochory or allochory. Finally, select behavior means that flora might exist or become endangered because of the climatic conditions.

\section{OBL strategy}

The OBLmodel is defined to be an effective optimization module developed by Tizhoosh for improving the convergence speed of diverse heuristic optimization methods [19]. The effective execution of OBLcontains estimation of opposite population as well as recent population in similar generation which helps in identifying best candidate solution of the provided problem. OBLis often employed in meta-heuristics approaches for enhancing the convergence speed.

Assume $N(N \in[x, y])$ be a real value. The opposite number $N^{\mathrm{O}}$ is described by

$$
N^{0}=x+y-N
$$

For d-dimensional search space, the description might be expanded as:

$$
N_{i}^{0}=x_{i}+y_{i}-N_{i}
$$

where $\left(N_{1}, N_{2} N_{d}\right)$ be the point in d-dimensional search space as given below.

$$
N_{i} \in\left[x_{i}, y_{i}\right] ;=\{1,2,3 d\} .
$$

OBL is applied for allinitialization stage with the help of generation jumping rate, $J_{\mathrm{r}}$.

\section{B. Proposed Fitness Function (FF)}

The FF of the OAF algorithm is derived by the use of 4 QoS metrics namely energy, link lifetime, congestion and distance [17]. Then, the route between the nodes is selected based on these measures. The major aim of the presented FF is to attain minimum FF value with maximum energy and link lifetime and minimum delay congestion and distance. The presented FF involves two main objective functions, as provided below. The FF of presented OAFmodels is developed with the help of 4 QoSparameters such as, energy, link duration, congestion control and distance. These 4 QoSparameters are applied while selecting the path in QoS aware routing protocol. The key objective of proposed FF is to reach lower FF value with higher energy, link lifetime, lower distance and congestion control distance. It is composed with 2 objectivefunctions which are expressed as:

$$
F_{f i \mathrm{t}}=\alpha\left[100-f_{o b j 1}\right]+\beta\left[f_{o b j 2}\right]
$$

where $f_{o b j 1}$ and $f_{o b j 2}$ are 2 objective functions; $a$ and $\mathrm{b}$ areweighted constant variables. The value of $\alpha$ is 0.1 while value of $\beta$ is 0.9 .

The FF presented is referred as minimization function. The factor with first objective function is reduced from 100. It is due to the value of $f_{o b j 1}$ that is linked with theenergy and the LLT constraints and produces higher value among 0 and 100 . It is accomplished by limiting the values from 100 which results in least function value. The factor with second objective function is comprised with congestion control and distance value.

\subsection{OAF Enabled Multipath Selection Process}

The presented OAF algorithm and the FF are employed in simulated MANET to select routes for data transmission between the mobile nodes. A brief explanation of the presented QCMRP-FF model is discussed in the subsequent subsections.

\section{A. Initialization}

First and foremost, the mobile nodes are placed in a particular MANET area. The connections among the nodes are used to 
make communication among the mobile nodes. Among two mobile nodes, the number of routes exists for communications between two nodes are explored.

\section{B. Route Selection}

From the available set of $\mathrm{K}$ routes for $\mathrm{S}$ and $\mathrm{D}$, optimum route is chosen based on OAF and FF. Among the identified $\mathrm{k}$ routes, a new route gets produced by the use of OAF model. The produced routes are assessed utilizing FF to choose the optimum paths. The presented FF is QoS aware, i.e. routes with optimum QoS are chosen as superlative route. An arbitrary route creation using OAF algorithm takes place till optimum route is identified. The number of solutions in the $\mathrm{OAF}$ algorithm is equivalent to the number of routes identified. The region of the identified route lies on the connection exists among the mobile nodes linked in the network. Among the identified path, a novel path gets produced by the use of OAF algorithm.

The range of the path discovered lies on the link existing between the mobile nodes associated with the network. From the discovered path, the new path is generated using the OAF algorithm. The recently produced solutions of the flora are validated by the $\mathrm{FF}$ and the previous solution gets replaced with the produced one, when the FF of the produced solution is minimal. In this case, the optimum route is chosen from the $\mathrm{K}$ identified routes.

\section{Stopping Criteria}

When the optimum number of routes was chosen, the OAF algorithm gets termination. The communication between two nodes takes place by the use of optimum routes with minimum congestion, maximum link lifetime and energy.

\section{PERFORMANCE VALIDATION}

The experimentation of the QCMRP-OAF algorithm is carried out using Network Simulator (NS 2.35) tool. The experiments were carried out under varying number of nodes and simulation time.

\subsection{Average residual energy Analysis}

Figure2 investigates the comparative results analysis of the QCMRP-OAF algorithm with respect to average residual energy under the existence of 50 and 100 nodes correspondingly. On determining the average residual energy under the application of various number nodes, the figure depicts that the QCMRP-OAF algorithm has reached efficient outcome by accomplishing maximum average residual energy than the earlier methods. It is evident that the AOMDV model is unfit to exhibit optimal results by reaching lower average residual energy compared to alternate models. Meanwhile, it is clear that the MMQARP, FCS-MQARP and CS-MQARP models have shown comparative average residual energy. Both the models have exhibited vital performance over the previous approaches by accomplishing gradual average residual energy. Hence, the projected QCMRP-OAF algorithm has implied best results than other technologies and achieved maximum average residual energy under varying simulation time.
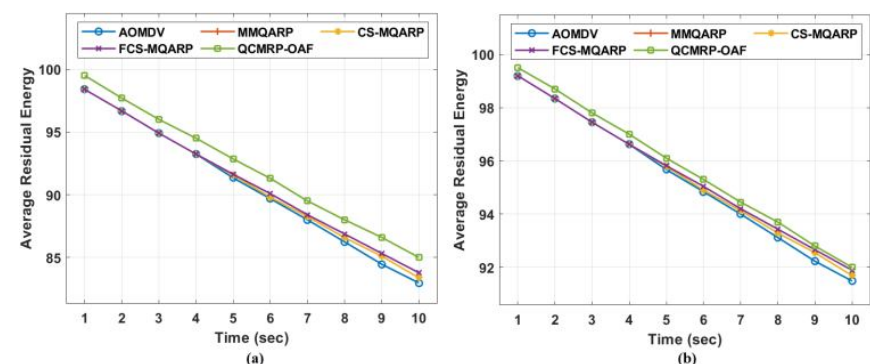

Figure 2: Performance Analysis of existing with proposed method based on Average Residual Energy a) 50 Nodes b) 100 Nodes

\subsection{Throughput Analysis}

Figure3 defines the comparative results analysis of the QCMRP-OAF algorithm by means of throughput under the existence of 50 and 100 nodes respectively. On calculating the throughput under the application of varying number of nodes, the figure reveals that the QCMRP-OAF algorithm has achieved productive outcome by obtaining higher throughput than the compared methods. It is noted that the MMQARP model is not suitable to provide manageable results by offering least throughput than other methods. Simultaneously, it is pointed that the CS-MQARP model tends to produce best results over MMQARP by attaining reasonable throughput.

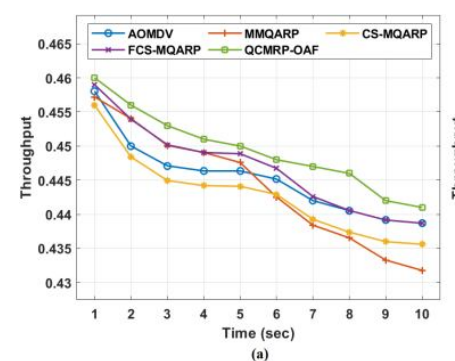

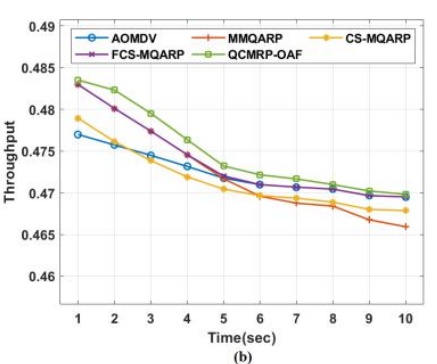

(b)
Figure 3: Performance Analysis of existing with proposed method based on Throughput a) 50 Nodes b) 100 Nodes

In line with this, the FCS-MQARP and AOMDV methods have shown equivalent throughput. These 2 models have implied effective performance over the previous models by obtaining gradual throughput. Thus, the newly proposed QCMRP-OAF algorithm has implemented successful outcome when compared alternate approaches by reaching greater throughput under diverse simulation time.

\subsection{Packet Loss Analysis}

Figure4 investigates the comparative results analysis of the QCMRP-OAF algorithm in terms of packet loss under the application of 50 and 100 nodes respectively. On determining the packet loss under the existence of diverse number of nodes, the figure states that the QCMRP-OAF method has accomplished efficient result by attaining least packet loss when related with other methodologies. It is pointed that the AOMDV model is not applicable for exhibiting target results 
by achieving maximum packet loss than other approaches. Meanwhile, it is pointed that the MMQARP algorithm tends to generate appreciable results than AOMDV by providing a minimum packet loss.
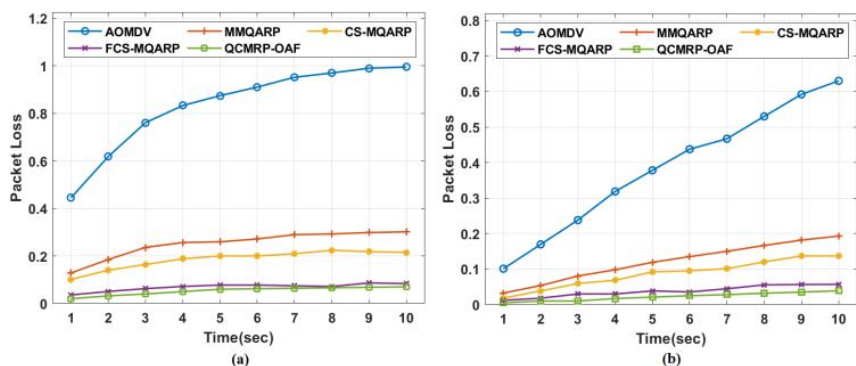

Figure 4: Performance Analysis of existing with proposed method based on Packet Loss a) 50 Nodes b) 100 Nodes

Along with that, the FCS-MQARP and CS-MQARP technologies have reached competing packet loss. These methods have demonstrated effective function when compared with existing schemes by reaching gradual packet loss. Hence, the newly proposed QCMRP-OAF algorithm has implied qualified results over the related models and achieved lower packet loss under different simulation time.

\subsection{Congestion Level Analysis}

Figure 5 describes the comparative results analysis of the QCMRP-OAF method with respect to congestion level under the existence of 50 and 100 nodes correspondingly. On evaluating the congestion level under the application of varying number of nodes, the figure points that the QCMRP-OAF algorithm has reached effective outcome by accomplishing less congestion level than the compared methods. It is apparent that the AOMDV model has failed to exhibit best results by achieving higher congestion level when compared to alternate methods. Concurrently, it is noticeable that the MMQARP model provides better outcome than AOMDV by attaining least congestion level. In line with this, the FCS-MQARP and CS-MQARP methodologies have shown more or less identical congestion level. Both the techniques have resulted significant function over the previous models by obtaining considerable congestion level. Therefore, the projected QCMRP-OAF algorithm has showcased qualified results over the earlier methods and reached lower congestion level under varying simulation time.
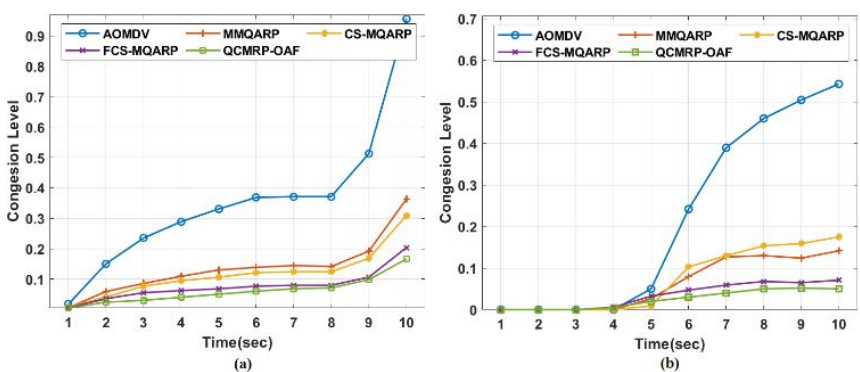

Figure 5: Performance Analysis of existing with proposed method based on Congestion Level a) 50 Nodes b) 100 Nodes

\section{CONCLUSION}

This paper has introduced a new QoS based Congestion Aware Multipath Routing Protocol using QCMRP-OAF algorithm. The application of OBL mechanism in AF algorithm helps to increase the convergence rate and exploration process. At the earlier stage, the mobile nodes in MANET undergo initialization in a particular target region. Next, the routes between any of the two nodes are identified. At last, the multiple routes are chosen by the QCMRP-OAF algorithm and derived FF. In addition, a new FF is also derived in QCMRP-OAF algorithm for route selection using energy, link lifetime, congestion, and distance. The experimental outcome indicated that the presented QCMRP-OAF algorithm is superior to other methods interms of average residual energy, throughput, delay, packet loss and congestion level. In future, the performance of the proposed model can be improved by the use of intrusion detection techniques.

\section{REFERENCES}

1. Ahmed. A. A. Gad-Elrab, Shereen A. El-aal, Neveen I. Ghali and Afaf A. S. Zaghrout, A Dynamic Genetic-Based Context Modeling Approach in Internet of Things Environments, International Journal of Advanced Trends in Computer Science and Engineering (IJATCSE), Volume 8 No. 6 (2019), pp. 2699 - 2709 https://doi.org/10.30534/ijatcse/2019/03862019

2. S. Mahalakshmi and Dr.R.Latha, Detection of single-trial EEG of the neural correlates of familiar faces recognition using machine-learning algorithms, International Journal of Advanced Trends in Computer Science and Engineering (IJATCSE), Volume 8 No. 6 (2019), pp. 2855 $-2860$ https://doi.org/10.30534/ijatcse/2019/28862019

3. Yung-Fa Huang, Jong-Shin Chen and Li-Ming Wang, Performance of Transmission Delay and Energy Consumption of Adaptive Listening Mechanism for Wireless Sensor Networks, International Journal of Advanced Trends in Computer Science and Engineering (IJATCSE), Volume 8 No. 6 (2019), pp. 3215-3220 https://doi.org/10.30534/ijatcse/2019/88862019

4. C. Perkins and P. Bhagwat, "Highly dynamic destinationsequenced distance-vector routing (DSDV) for mobile computers ," in Proceedings of the ACM Conference on Communications Architectures, Protocols and Applications (SIGCOMM '94), October 1994.

5. S. Murthy and J. J. Garcia-Luna-Aceves, "Routing protocol for packet radio networks," in Proceedings of the 1st Annual International Conference on Mobile Computing and Networking (MobiCom '95), pp. 86-95, Berkeley, Calif, USA, November 1995.

6. T. G. Basavaraju, S. K. Sarkar, C. Puttamadappa, and M. A. Gautham, "ECARP: an efficient congestion adaptive routing protocol for mobile Ad hoc networks," in Proceedings of the 6th International Conference on ITS Telecommunications Proceedings, pp. 715-718, Chengdu, China, June 2006. 
R. Jayaraj et al., International Journal of Advanced Trends in Computer Science and Engineering, 9(4), July - August 2020,5121 - 5127

7. X. Chen, H. M. Jones, and A. D. S. Jayalath, "Congestion-aware routing protocol for mobile ad hoc networks," in Proceedings of IEEE Conference of Vehicular Technology, pp. 21-25, October 2007.

8. S. Sharma, R. C. Jain, and S. S. Bhadauria, "Simulation study of congestion adaptive routing algorithm for mobile ad hoc network," Trends in Applied Sciences Research, vol. 1, pp. 368-378, 2006.

9. S. S. Baboo and B. Narasimhan, "A hop-by-hop congestionaware routing protocolfor heterogeneous mobile ad-hoc networks," International Journal of Computer Science and Information Security, vol. 3, no. 1, 2009.

10. R. Hashim, Q. Nasir, and S. Harous, "Congestion aware multipath dynamic source routing protocol (CAWMP-DSR) for mobile Ad-Hoc network," in Proceedings of the 5th International Conference on Advances in Mobile Computing and Multimedia (MoMM '07), Jakarta, Indonesia, December 2007.

11. A. Valarmathi and R. M. Chandrasekaran, "Congestion aware and adaptive dynamic source routing algorithm with loadbalancing in MANETs," International Journal of Computer Applications, vol. 8, no. 5, article 1, 2010. https://doi.org/10.5120/1210-1734

12. A. Gaba, S. Voulgaris, and M. van Steen, "Towards congestionaware all-to-all information dissemination in mobile ad-hoc networks," in Proceedings of the 2010 IEEE Globecom Workshops (GC '10), pp. 1690-1695, Miami, Fla, USA, December 2010.

13.X. Chen, H. M. Jones, and A. D. S. Jayalath, "Congestion-aware routing protocol for mobile ad hoc networks," in Proceedings of the IEEE 66th Vehicular Technology Conference (VTC '07), pp. 21-25, Baltimore, Md, USA, October 2007.

14. B. Ramesh and D. Manjula, "CA-AODV: congestion adaptive AODV routing protocol for streaming video in mobile Ad Hoc networks," I. J. Communications, Network and System Sciences, vol. 4, pp. 285-385, 2008.

15. Y. J. Lee and G. F. Riley, "A workload-based adaptive loadbalancing technique for mobile ad hoc networks," in Proceedings of the IEEE Wireless Communications and Networking Conference (WCNC '05), vol. 4, pp. 2002-2007, March 2005.

16. S. Soundararajan and R. S. Bhuvaneswaran, "Adaptive multipath routing for load balancing in mobile Ad Hoc networks," Journal of Computer Science, vol. 8, no. 5, pp. 648-655, 2012. https://doi.org/10.3844/jcssp.2012.648.655

17. Rama Rao A., Satyananda Reddy, ValliKumari V., (2018) "Multi-path selection based on fractional cuckoo search algorithm for QoS aware routing in MANET", Sensor Review, https://doi.org/10.1108/SR-08-2017-0170

18. Cheng, L., Wu, X.H. and Wang, Y., 2018. Artificial flora (AF) optimization algorithm. Applied Sciences, 8(3), p.329.

19. Tizhoosh, H.R. and Ventresca, M. eds., 2008. Oppositional concepts in computational intelligence (Vol. 155). Springer.

https://doi.org/10.1007/978-3-540-70829-2 